\title{
gC-Studio - the environment for automated filter design
}

\author{
P. KATARZYNSKI*, M. MELOSIK, and A. HANDKIEWICZ \\ Computer Engineering, Poznan University of Technology, Piotrowo 3A, 60-965 Poznań, Poland
}

\begin{abstract}
The paper presents the idea of software suite integrating the tools supporting the analog filter design. It uses the prototype circuits that are composed of gyrators and capacitors. The essential, behavioral parameters are characterised for the filtering structures. The basic assumptions formulated before the implementation are also mentioned. The structure of the software suite is discussed, its functional properties and the implementation issues are mentioned. The resulting software brings the automation of designing SISO filters as well as the filter pairs. In the proposed solution the VHDL-AMS language is assumed as the formal method of hardware description.
\end{abstract}

Key words: gyrator-capacitor prototype circuits, filters approximations, EDA, CAE.

\section{Introduction}

Filtering is the most basic operation applied to electrical signals. It helps to efficiently remove the unwanted components such like noise or distortions. The filtering process may be utilized also for choosing the useful data from the analyzed signal. Considering the miniaturization of electronic devices, especially those oriented for mobile and wireless communication there appears the necessity of developing design methodologies that ensure complex realization of particular filtering structures. The manifestation of such approach is the idea of silicon compilers that will give the opportunity of fully automated fabrication for mixed signal integrated chips.

In recent years the inductance-capacitor (LC) passive circuits have been used for filtering purposes as they are known for their decent selectivity. Therefore LC structures are suitable signal processing in wireless communications as well as for filtering in power electronics [1-2]. Nevertheless, implementing the inductances onto integrated chip proves to be tricky [3-4]. Such an approach involves employing sophisticated and thus expensive chip manufacturing techniques. However, it is possible to express the topology of LC circuits in terms of so called prototype circuits composed of gyrators and capacitors [5]. Figure 1 presents the example of LC structure being the low-pass elliptic filter compared to its gyrator-capacitor counterpart. Introducing the gyrators for simulating the inductances is a common practice utilized mainly in power electronic devices such as the impulse power supplies or voltage converters [6]. It is also important that nonreciprocal $\mathrm{gC}$ circuits are able to realize wider class of transfer functions in comparison to simple LC ladder structures [5]. The gyrator-capacitor structure formulates the prototype circuit that may be further realized as integrated chip by using the standard CMOS fabrication process [7]. For scientific purposes the Matlab is commonly used in the process of analog and digital filter design [8]. However, due to the relatively high complexity of filtering structures we observe the strong need for developing computer software that speeds up and simplifies the whole design process following the methodology of Electronic Design Automation (EDA) [9]. Similar EDA oriented issues regarding different filtering structures (such like active RC filters) are investigated extensively nowadays [10] which in consequence may bring substantial progress in the rapid design, simulation and manufacturing of analogue and mixed signal integrated circuits.

a)

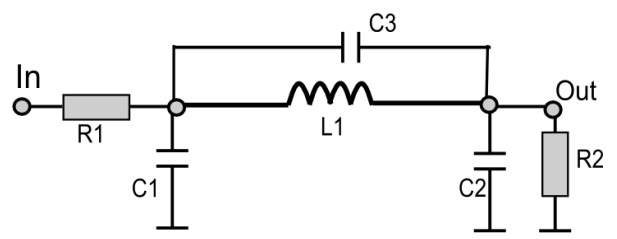

b)

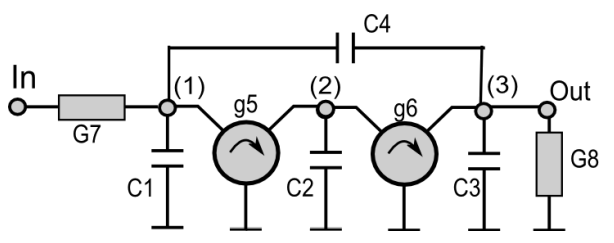

Fig. 1. Ladder structure of the LP elliptic filter (a) with gyratorcapacitor equivalent (b)

\section{Behavioral description for filtering purposes}

The filtering process may be uniquely defined by providing the mathematical model in a form of the transfer function:

$$
H(s)=h_{0} \frac{\prod_{j=0}^{J}\left(s-z_{j}\right)}{\prod_{k=1}^{K}\left(s-p_{k}\right)} J \leq K,
$$

where $z_{j}, p_{k}$ are complex numbers referring to zeroes and poles onto the complex plane. They determine the properties of frequency response for the given filtering structure. Hence the transfer function modeling is in fact the process of finding the values for subsequent zeroes and poles iteratively [11]. The

\footnotetext{
*e-mail: piotr.katarzynski@put.poznan.pl
} 
frequency response of the filter with a given transfer function may be characterized by so called behavioral parameters. On such basis we can consider the following:

- $A_{p}$ ripple amount in the pass-band (PB);

- $A_{s}$ minimal attenuation in the stop-band (SB);

- $w_{p}$ boundary angular frequency starting the transitional band (TB);

- $w_{s}$ boundary angular frequency ending the transitional band (TB).

Our realization of the EDA software suite for supporting filter design started from implementing the algorithms that generate the commonly used approximations of transfer functions. Such numerical methods require the $\left\{A_{p}, A_{s}, w_{p}, w_{s}\right\}$ parameters as the input data. Then the next step in the design process would require refinement of appropriate prototype circuit, which provides the same frequency response as the considered mathematical model given in a form of the transfer function.

From the designer's point of view it is important to ensure the unified way of describing the topologies of circuits under design. Such feature is realized by using the hardware description languages and the approach is nowadays the common practice in the digital electronics. It is enough to mention here the VHDL or Verilog that for analogue domain evolved to VHDL-AMS or Verilog-A. It is worth to stress, however, that the analogue versions of the mentioned languages are not synthesizable in general and thus they are used mainly for simulation purposes [12]. In terms of the considered EDA software the VHDL-AMS language was proposed as the way of expressing the topologies of gyrator-capacitor structures. Figure 2 presents the example of the prototype circuit with the corresponding HDL description. The process of describing the gyrator-capacitor structures $(\mathrm{gC})$ in our software should conform to some basic guidelines that refer to the general VHDL-AMS syntax:

- the design entity must have the grounded terminal and at least one terminal for input (excitation) and one for output (response) signals that are assumed to be voltages with respect to given grounded reference node;

- the topology of the circuit may be decomposed into a subset of functional blocks represented by instances of virtual component GC_BLOCK;

- the content of each instance representing the GC_BLOCK may be parameterized by using the VHDL generic statements. Connections between blocks and the external signals are defined by using the HDL port mapping syntax;

- currently only one architecture may be associated with a given design entity within a single VHDL-AMS file.

Figure 3 presents the structure of a single $G C \_B L O C K$. It is spanned between internal nodes of the circuit $N_{i}, N_{j}$. In a single instance of the block the $C_{i}, C_{j}, C_{f}$ capacitances and $i g$ gyrator are optional. Their presence is controlled by generic map statements. Nodes $N_{i}$ and $N_{j}$ may be connected to external signals (these appear as ports in the entity declaration). External input signals connected directly to the GC_BLOCK enhance it with series conductance $\left(G_{i 1}\right.$ or $\left.G_{j 1}\right)$ whereas the external output signals introduce the parallel conductances $\left(G_{i 2}\right.$ or $\left.\mathrm{G}_{j 2}\right)$.

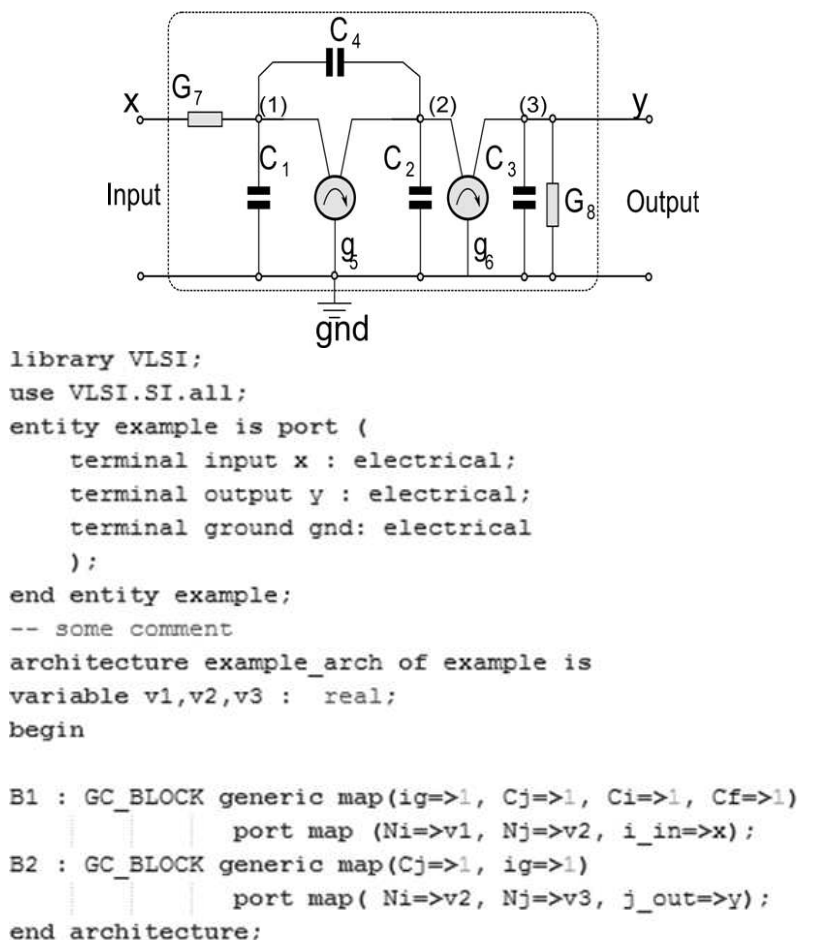

Fig. 2. Example of the $\mathrm{gC}$ structure and its VHDL-AMS description

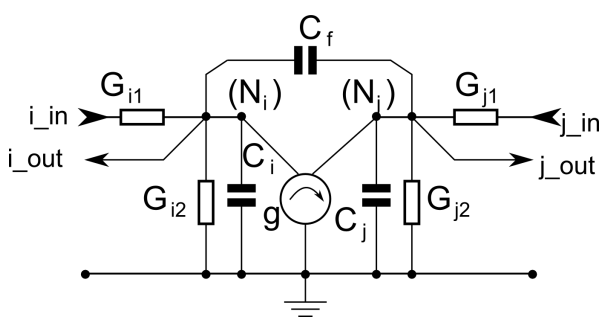

Fig. 3. Structure of the elementary block used for circuit's topology description

The VHDL-AMS files that define the filtering structures may be easily parsed by software tools for extracting the topological information. This leads to formulate the netlist of the components. After this stage the netlist may be used for topological analysis. On that basis it is possible to obtain the transfer function expressed in a symbolic form. This implies that the polynomials of the numerator and denominator, which appear in symbolic transfer function, have their coefficients expressed as linear combinations of symbolic variables that represent the values of the components used in the circuit. In order to provide the efficient way of storing and processing the symbolic forms of transfer functions, the structural numbers have been used [13]. More details concerning this issue may be found in [14].

The synthesis of gyrator-capacitor filter requires estimating the values for parameters of capacitances, gyrations and conductances so the circuit treated as a set of such components connected together will produce the expected frequen- 


\section{$g C$-Studio - the environment for automated filter design}

cy response. This involves comparison between mathematical (calculated as approximation) and symbolic form of the transfer function. Finding the values of parameters simplifies to the issue of solving a set of non-linear equations. In the EDA software the Hooke and Jeeves direct search algorithm was implemented.

Several topics have been put into consideration when comes to the functionality of the proposed EDA software. It is enough to mention the automated maintenance of the project's design life cycle. The second major thing is the modularity so particular applications included in the EDA software suite may be further developed independently from each other.

\section{Results}

The $g C$-Studio software suite integrated several applications:

- gc_analyser, that creates the netlist of elements basing onto the model provided with VHDL-AMS syntax. It constructs the symbolic matrices for the nodal potential method and calculates their determinants in order to provide the symbolic transfer function. It may also export the data at any stage of the analysis to the third party software such like Matlab;

- filter, that finds the mathematical approximations of the transfer functions for filters of given properties for their frequency responses. Currently the Butterworth, Chebyshev and Cauer approximations are supported. Both low-pas and high-pass versions are obtainable. Moreover, the software is capable of producing the transfer functions for filter pairs [15];

- param_calc, that implements the Hooke-Jeeves direct search algorithm in order to find the values of the components in the circuit;

- gc_sim, simulates the frequency responses for both symbolic and approximated transfer functions. It is also possible to realize the sensitivity analysis with respect to values of parameters.

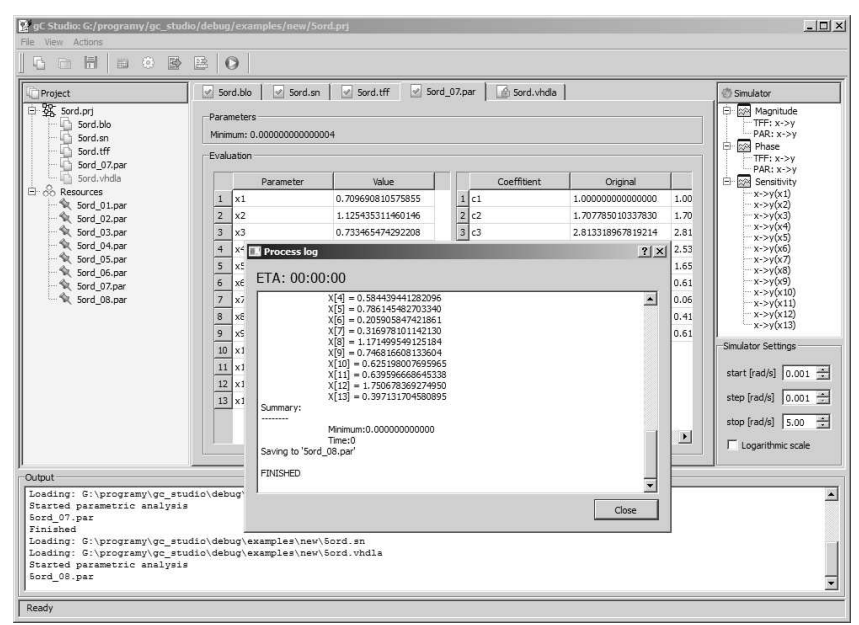

Fig. 4. Main application's window with launched process of analysis

The main window of application (Fig. 4) has the appearance and layout that correspond to typical software oriented project management. At the left side of the window there is a tree-like structure that represents the project with associated data files. The central part of the window contains a tabbed view that presents the content of particular project files. The right side of the window contains the visual controls that allow altering the simulator settings.

\section{Summary}

The obtained software suite supports the behavioral synthesis of filtering structures that in general may be multiport circuits. According to filter pairs the mathematical approximation for the transfer functions have been implemented basing onto the elliptic low-pass filter. Figures 5 and 6 present the frequency responses of filter pairs obtained with $g c_{-}$sim as well as their HSpice counterparts. The results presented for $g C$-Studio contain the responses before and after the circuit synthesis. The proposed approach has given us the opportunity to implement EDA software suite. In consequence it helped to make VHDL-AMS language synthesizable for the class of discussed filtering circuits. The strength of the elaborated design environment is illustrated in the filter pair example. The compact $\mathrm{gC}$ circuit structure allows obtaining OTA-C, SI or SC counterpart filter pairs with minimized number of active elements (transconductance or operational amplifiers) in comparison to design methods elaborated up to now.

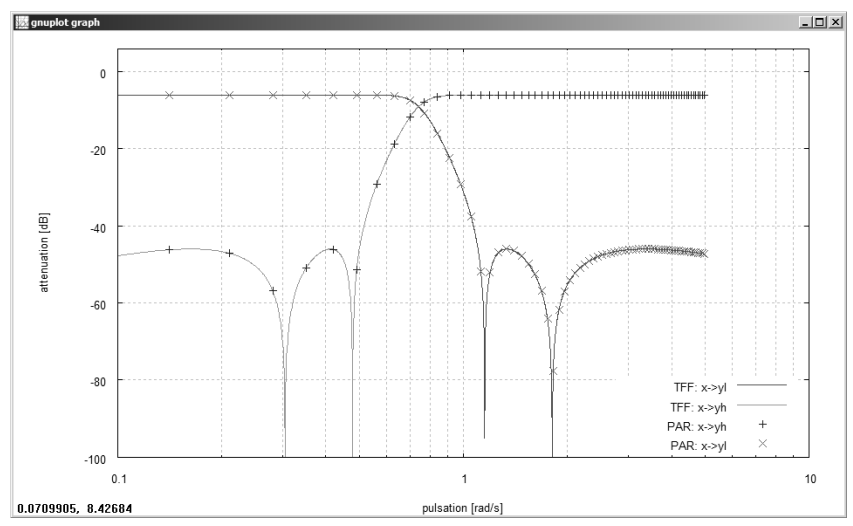

Fig. 5. Frequency responses for filter pair obtained with $g C$-Studio (TFF - mathematical model, PAR - parametric analysis)

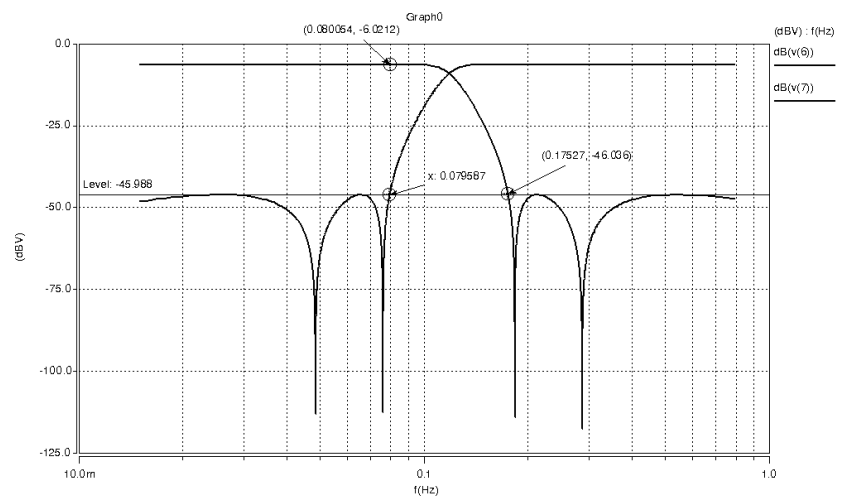

Fig. 6. Frequency response of gyrator-capacitor model for the filter pair obtained with HSpice 
P. Katarzynski, M. Melosik, and A. Handkiewicz

\section{REFERENCES}

[1] P. Mysiak and R. Strzelecki, "A robust 18-pulse diode rectifier with coupled reactors", Bull. Pol. Ac.: Tech. 55 (4), 541-550 (2007).

[2] M. Siwczyński and M. Jaraczewski, "Reactive compensator synthesis in time-domain", Bull. Pol. Ac.: Tech. 60 (1), 119124 (2012)

[3] B.J. Pierquet, T.C. Neugebauer, and D.J. Perreault, "A fabrication method for integrated filter elements with inductance cancellation", IEEE Trans. on Power Electronics. 24 (3), 838848 (2009).

[4] P.J. Sullivan, B.A. Xavier, and W.H. Ku, "An integrated CMOS distributed amplifier utilizing packaging inductance", IEEE Trans. on Microwave Theory and Techniques 45 (10), 19691976 (1997).

[5] A. Handkiewicz, Mixed-Signal Systems. A Guide to CMOS Circuit Design, Wiley-IEEE Press, London, 2002.

[6] D.C. Hamill, "Lumped equivalent circuits of magnetic components: the gyrator-capacitor approach", IEEE Trans. on Power Electronics 8 (2), 97-103 (1993).

[7] F. Yuan, "CMOS gyrator-C active transformers", Circuits, Devices \& Systems, IET 1 (6), 494-508 (2007).

[8] A. Slowik and M. Bialko, "Design of IIR digital filters with non-standard characteristics using differential evolution algorithm”, Bull. Pol. Ac.: Tech. 55 (4), 359-363 (2007).

[9] S. Krolikoski, "Evolution of EDA standards worldwide", IEEE Design \& Test of Computers 28 (1), 72-75 (2011).

[10] S. Koziel and S. Szczepanski, "General active-RC filter model for computer-aided design”, Bull. Pol. Ac.: Tech. 54 (1), 89-99 (2006).

[11] C. B. Rorabaugh, DSP Primer, McGraw-Hill, London, 1998

[12] F. Pecheux, C. Lallement, and A. Vachoux, "VHDL-AMS and Verilog-AMS as alternative hardware description languages for efficient modeling of multidiscipline systems", IEEE Trans. on Computer-aided Design of Integrated Circuits and Systems 24 (2), 204-225 (2005).

[13] S. Bellert, "Topological analysis and synthesis of linear systems", J. Franklin Institute 274 (6), 425-443 (1962).

[14] P. Katarzynski, M. Melosik, M. Naumowicz, and S. Szczesny, "Symbolic analysis in gyrator-capacitor filters", Mixed Design of Integrated Circuits and Systems (MIXDES), 2012 Proc. 19th Int. Conf. 1, 392-397 (2012).

[15] A. Handkiewicz, P. Katarzyński, S. Szczęsny, J. Wencel, and P. Śniatała, "Analog filter pair design on the basis of a gyratorcapacitor prototype circuit", Int. J. Circuit Theory and Applications 40 (6), 539-550 (2012). 